\title{
Corrigendum
}

\section{Nanotomography of Inverse Photonic Crystals Using Zernike Phase Contrast - CORRIGENDUM}

Silja Flenner, Emanuel Larsson, Kaline Pagnan Furlan, Daniel Laipple, Malte Storm, Fabian Wilde, Robert Blick, Gerold A. Schneider, Robert Zierold, Rolf Janssen, Christian David, Felix Beckmann, Martin Müller and Imke Greving

Doi: doi.org/10.1017/S1431927618013120, Published by Cambridge University Press, 10 August 2018

In the original publication, author Kaline Pagnan Furlan's name was incomplete. The full author name appears correctly above.

\section{Reference}

Flenner S, Larsson E, Furlan KP, Laipple D, Storm M, Wilde F, Blick R, Scheider GA, Zierold R, Janssen R, David C, Beckmann F, Müller M and Greving I (2018) Nanotomography of Inverse Photonic Crystals Using Zernike Phase Contrast. Microsc Microanal 24(S2), 146-147. doi. org/10.1017/S1431927618013120. 